\title{
Evolución de la estructura demográfica municipal en el departamento de Boyacá 1964 2005
}

Evolution of the municipal demographic structure in the Colombian department of Boyacá, 1964 2005

\section{María Alexandra Gutiérrez Vera ${ }^{1}$}

\section{Resumen}

La dinámica demográfica de los municipios boyacenses entre 1964 y 2005 presenta importantes cambios en su estructura, los cuales se analizan en este documento por medio de diferentes índices demográficos municipales. Con este análisis se espera contribuir a su entendimiento y brindar información necesaria para identificar los lineamientos y tendencias en temas de planificación y organización del territorio.

Palabras clave: Boyacá, estructura de la población, planificación territorial.

\section{Abstract}

Demographic dynamics of Boyacá municipalities between 1964 and 2005 present important changes in its structure, which are discussed in this document through different demographic rates. With this analysis, we expect to contribute to the understanding and provide the needed information to identify the guidelines and trends in planning and organization of the territory.

Keywords: Boyacá, population structure, territorial planning. 


\section{Introducción}

La Geografía como ciencia que estudia y localiza los hechos y fenómenos físicos, biológicos y humanos que ocurren sobre la superficie terrestre, las causas que los originan y sus relaciones mutuas (Martonne, 1934), incluye dentro de sus ramas a la geografía Humana que ofrece una visión amplia y sinóptica de las relaciones espaciales en los asuntos humanos (Smith, 1980), y se encarga de estudiar la organización del espacio dada por la interrelación entre el hombre, sus actividades y el espacio mismo. La geografía de la población, como parte de la geografía humana, estudia las dinámicas demográficas y su diferenciación en el espacio como uno de sus temas de mayor importancia.

El presente trabajo, que se basa en el análisis de la información de los censos de población de 1964, 1973, 1985, 1993 y 2005 realizados por el Departamento Administrativo Nacional de Estadística DANE, pretende mostrar la evolución de la estructura poblacional de los municipios del departamento de Boyacá mediante el estudio de diferentes índices demográficos que facilitan identificar tendencias municipales, como los de envejecimiento, de dependencia y de renovación de población activa. Debido a la falta de disponibilidad de datos de natalidad confiables para años anteriores a 1993, se recurrió al uso de otros indicadores, como los índices de tendencia y de maternidad, que permiten vislumbrar el panorama general de fecundidad y natalidad en situaciones como la señalada (Vinuesa \& Francisco, 1997).

\section{Estructura de la población en Boyacá}

La estructura de la población de Boyacá entre 1964 y 2005 ha seguido un proceso de transformación demográfica caracterizado por la disminución de la población infantil, un aumento de la población joven y un ligero aumento de la cúspide de la pirámide (Figura 1).

Es decir, Boyacá pasó de un perfil demográfico expansivo en 1964 a un perfil regresivo en el 2005. Así mismo, las pirámides también evidencian el equilibrio entre población femenina y masculina en el departamento.

La constante disminución de la tasa general de fecundidad, sumada a la tasa de mortalidad infantil (Tabla 1) son algunas de las razones de la disminución de población infantil. 
Figura 1. Pirámides de población Boyacá 1964 -2005
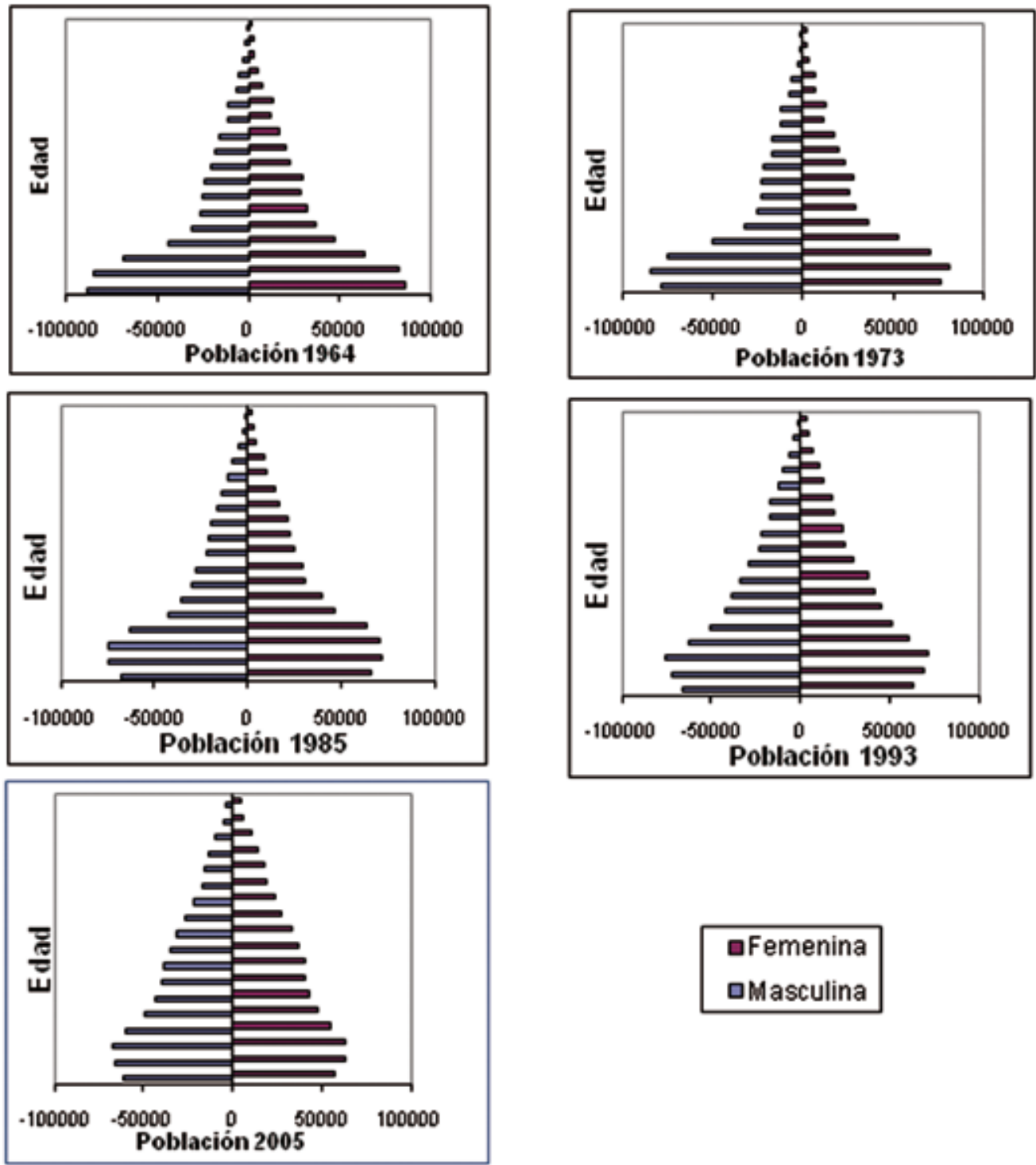

\section{口Femenina \\ 口Masculina}

Fuente: elaboración propia. Datos censos DANE 1964, 1973, 1985, 1993, 2005 
Tabla 1. Tasas de mortalidad infantil y fecundidad. Boyacá 1985- 2005

\begin{tabular}{|c|c|c|c|c|c|c|c|}
\hline \multicolumn{3}{|c|}{ TASA DE MORTALIDAD INFANTIL (por mil) } & \multicolumn{3}{c|}{ TASA GENERAL DE FECUNDIDAD (por mil) } \\
\hline $1985-1990$ & $1990-1995$ & $1995-2000$ & $2000-2005$ & $1985-1990$ & $1990-1995$ & $1995-2000$ & $2000-2005$ \\
\hline 37,00 & 35,70 & 31,80 & 27,40 & 117,00 & 109,70 & 99,80 & 90,20 \\
\hline
\end{tabular}

Fuente: DANE (2007)

Esta disminución paulatina de tasas de fecundidad, según Flórez (2000), se debe a los avances realizados en actividades enfocadas hacia la planificación familiar consolidados en la década del setenta, especialmente por Profamilia y el Ministerio de Salud, así como por el acceso a la educación por parte de la mujer.

La constricción de la pirámide en edades productivas es una señal de migración, que se aprecia en las altas tasas de migración de 1968-1973, en los grupos de edades medias, especialmente en los rangos de 20-24 y 25-29 con un 52\% y $40 \%$ respectivamente, las cuales no han presentado cambios sustanciales entre 1973 y 1993 (Martínez, 2006).

Es evidente que aunque la proporción de población mayor a 65 años ha sido baja, ha aumentado paulatinamente, pero especialmente en el 2005, lo cual puede manifestarse en un ligero aumento en la esperanza de vida (Tabla 2).
En 1964, la estructura demográfica en los municipios del departamento presentaba una distribución homogénea, todos los municipios de Boyacá tenían un perfil expansivo (Figura 2a), la base de la pirámide era ancha y disminuía a medida que aumentaba el rango de edad, pero esta estructura fue cambiando en la mayoría de los municipios, hacia una estructura regresiva (Tabla 3) caracterizada por una base angosta. Es así como en 1973 aparecen unos enclaves territoriales con estructura regresiva (Figura $2 b$ ) y ya en 1985 esta era la estructura dominante en el territorio boyacense (Figura 2c), aunque algunos municipios han permanecido con una estructura expansiva, especialmente aquellos que están ubicados en las zonas limítrofes del departamento (Figuras 2d y 2e).

Tabla 2. Esperanza de vida 1985- 2005

\begin{tabular}{|c|c|c|c|c|}
\hline & \multicolumn{4}{|c|}{ ESPERANZA DE VIDA (años) } \\
\hline & $\mathbf{1 9 8 5 - 1 9 9 0}$ & $\mathbf{1 9 9 0 - 1 9 9 5}$ & $\mathbf{1 9 9 5 - 2 0 0 0}$ & $\mathbf{2 0 0 0 - 2 0 0 5}$ \\
\hline Boyacá & 69,27 & 69,91 & 71,36 & 72,93 \\
\hline
\end{tabular}

Fuente: DANE (2007) 
Tabla 3. Distribución porcentual de municipios según estructura poblacional 1964 -2005

\begin{tabular}{|c|c|c|c|c|c|}
\hline & \multicolumn{5}{|c|}{ Municipios (\%) } \\
\hline Estructura & $\mathbf{1 9 6 4}$ & $\mathbf{1 9 7 3}$ & $\mathbf{1 9 8 5}$ & $\mathbf{1 9 9 3}$ & $\mathbf{2 0 0 5}$ \\
\hline Expansiva & 100 & 72 & 26 & 14 & 18 \\
\hline Estacionaria & 0 & 0 & 0 & 0 & 2 \\
\hline Regresiva & 0 & 28 & 74 & 86 & 80 \\
\hline
\end{tabular}

Fuente: elaboración propia

Algunos índices ayudan a explicar las tendencias en la estructura de la población analizadas anteriormente, entre ellos están el índice de tendencia, el de maternidad, de envejecimiento, de dependencia y el índice de renovación de población activa.

Figura 2. Evolución de la estructura poblacional 1964-2005

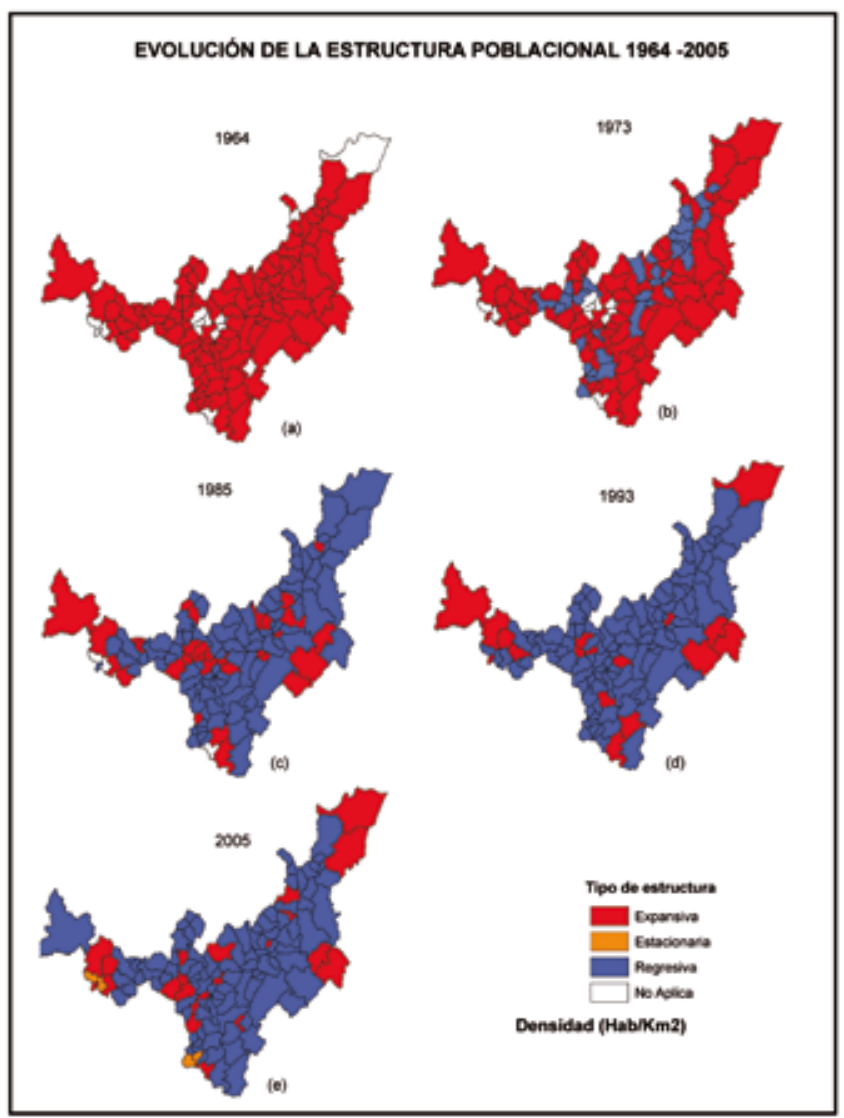

Fuente: elaboración propia. Cálculos basados en censos, DANE, 1964, 1973, 1985, 1993, 2005. 
El índice de tendencia se define como un indicador de la dinámica demográfica que a medida que presente valores inferiores a 100 estará reflejando descenso de la natalidad, menor crecimiento demográfico y envejecimiento. Este indicador presenta en 1964 un comportamiento diferente en los municipios del departamento comparado con el de 2005. En 1964, en el centro del departamento predomina un índice negativo (Figura 3a), mientras que en el resto del departamento prevalece un índice positivo (valores $\geq 100$ ), reflejando entonces un mayor crecimiento demográfico. Pero a partir de 1973 esta situación se revierte, y se expande la zona con índice negativo a tal punto de predominar en casi todo el territorio, patrón que se mantiene hasta 1993 (Figuras 3b, 3c), en el que solo el $11 \%$ de los municipios mantenían un índice positivo (Tabla 4) los cuales se localizan en los extremos limítrofes del departamento (Figura 3d). En el 2005 la distribución es similar, aunque unos municipios presentan recuperación del índice (Figura 3e) ya que el $19 \%$ de los municipios presenta nuevamente valores positivos.
Así mismo, el índice de maternidad, definido como la relación entre la población menor de cinco años y la población femenina en edad fértil, ofrece una aproximación a la fecundidad, lo que es de utilidad en caso de no contar con datos de natalidad confiables, como es el caso de Boyacá. En 1964 y 1973 se observa una distribución homogénea del índice de maternidad medio y algunos valores altos en municipios dispersos (Figuras 4a y 4b). En 1964, el 94 \% de los municipios experimentaba valores medios, situación similar en 1973 cuando el $90 \%$ de los municipios presentaba el mismo patrón. En 1985 aparecen grupos de municipios con valores bajos (Figura 4c), y en 1993 continúa la expansión de estos territorios con valores bajos, aunque el $50 \%$ de los municipios mantiene valores medios, de los cuales unos están dispersos en la zona occidental y otros agrupados en la franja oriental del departamento (Figura 4d). Ya en el 2005 son los municipios con índice bajo aquellos que representan la mayoría (77 \%) del departamento (Tabla 5), mientras que los municipios limítrofes de la zona nororiental y noroccidental aún presentan índices de maternidad medios

Tabla 4. Distribución porcentual de municipios según índice de tendencia, 1964-2005

\begin{tabular}{|c|c|c|c|c|}
\hline \multirow{2}{*}{ Periodo } & \multicolumn{2}{|c|}{ Negativo } & \multicolumn{2}{c|}{ Positivo } \\
\cline { 2 - 5 } & Num. Mpio & \% Mpio & Num. Mpio & \% Mpio \\
\hline 1964 & 35 & 32 & 75 & 68 \\
\hline 1973 & 91 & 80 & 23 & 20 \\
\hline 1985 & 92 & 76 & 29 & 24 \\
\hline 1993 & 109 & 89 & 14 & 11 \\
\hline 2005 & 104 & 85 & 19 & 15 \\
\hline
\end{tabular}

Fuente: elaboración propia. Cálculos basados en datos DANE, censos 1964, 1973, 1985, 1993, 2005 
Figura 3. Evolución del índice de tendencia. Boyacá 1964-2005

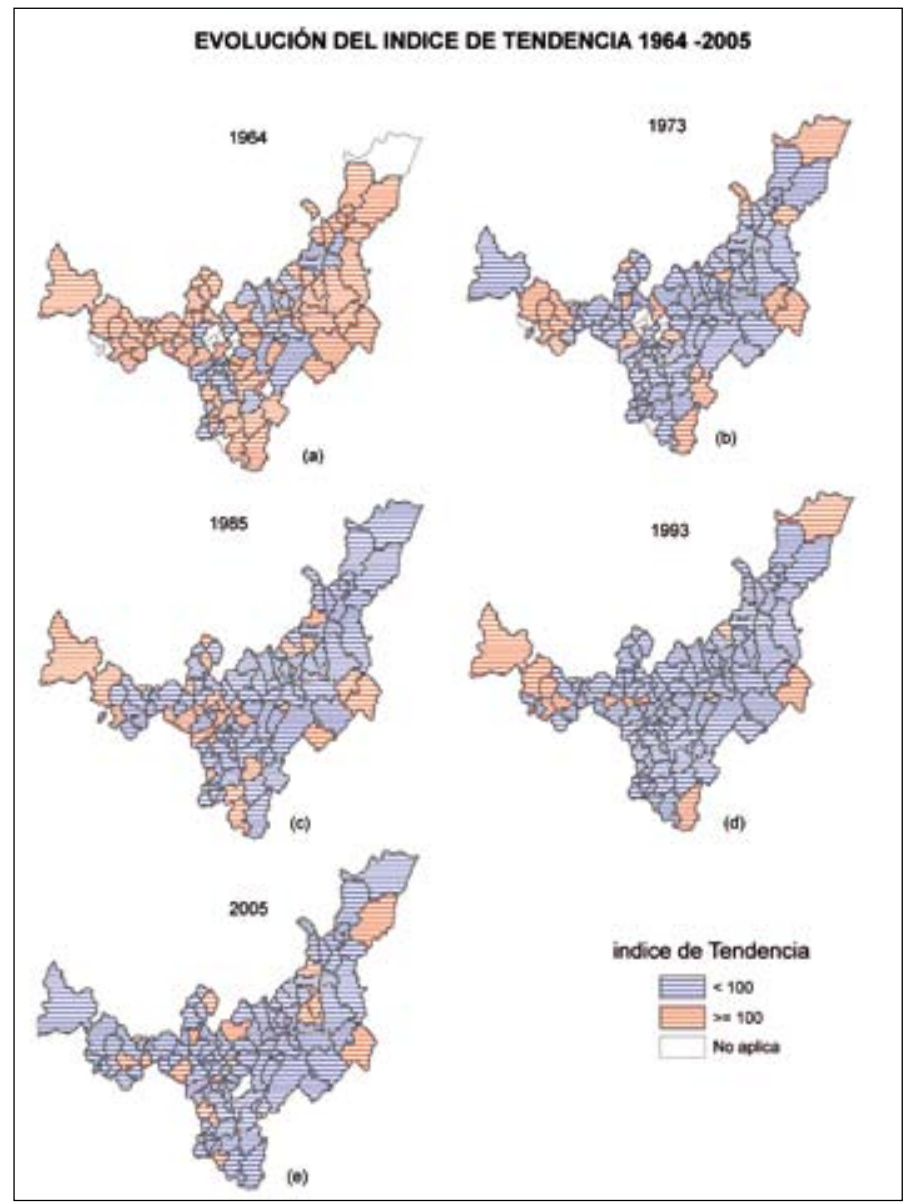

Fuente: elaboración propia. Cálculos basados en datos del DANE, censos 1964, 1973,1985, 1993, 2005.

Tabla 5. Distribución porcentual de municipios según índice de maternidad 1964-2005

\begin{tabular}{|c|c|c|c|c|c|c|}
\hline \multirow{2}{*}{ Periodo } & \multicolumn{2}{|c|}{ Bajo } & \multicolumn{2}{c|}{ Medio } & \multicolumn{2}{c|}{ Alto } \\
\cline { 2 - 7 } & Num. Mpio & \% Mpio & Num. Mpio & \% Mpio & Num. Mpio & \% Mpio \\
\hline 1964 & 0 & 0 & 103 & 94 & 7 & 6 \\
\hline 1973 & 1 & 1 & 103 & 90 & 10 & 9 \\
\hline 1985 & 39 & 32 & 81 & 67 & 1 & 1 \\
\hline 1993 & 62 & 50 & 61 & 50 & 0 & 0 \\
\hline 2005 & 95 & 77 & 28 & 23 & 0 & 0 \\
\hline
\end{tabular}

Fuente: elaboración propia. Cálculos basados en censos, DANE, 1964, 1973, 1985, 1993, 2005 
(Figura 4e). Se observa entonces que la evolución de este índice muestra una tendencia a la disminución de la maternidad, reflejándose en la constricción de la población infantil y por ende en un menor crecimiento demográfico hacia el futuro.
En ese orden de ideas es importante investigar las causales de disminución de las tasas de maternidad en los municipios con valores bajos y emprender acciones necesarias para evitar un futuro despoblamiento.

Figura 4. Evolución del índice de maternidad 1964-2005

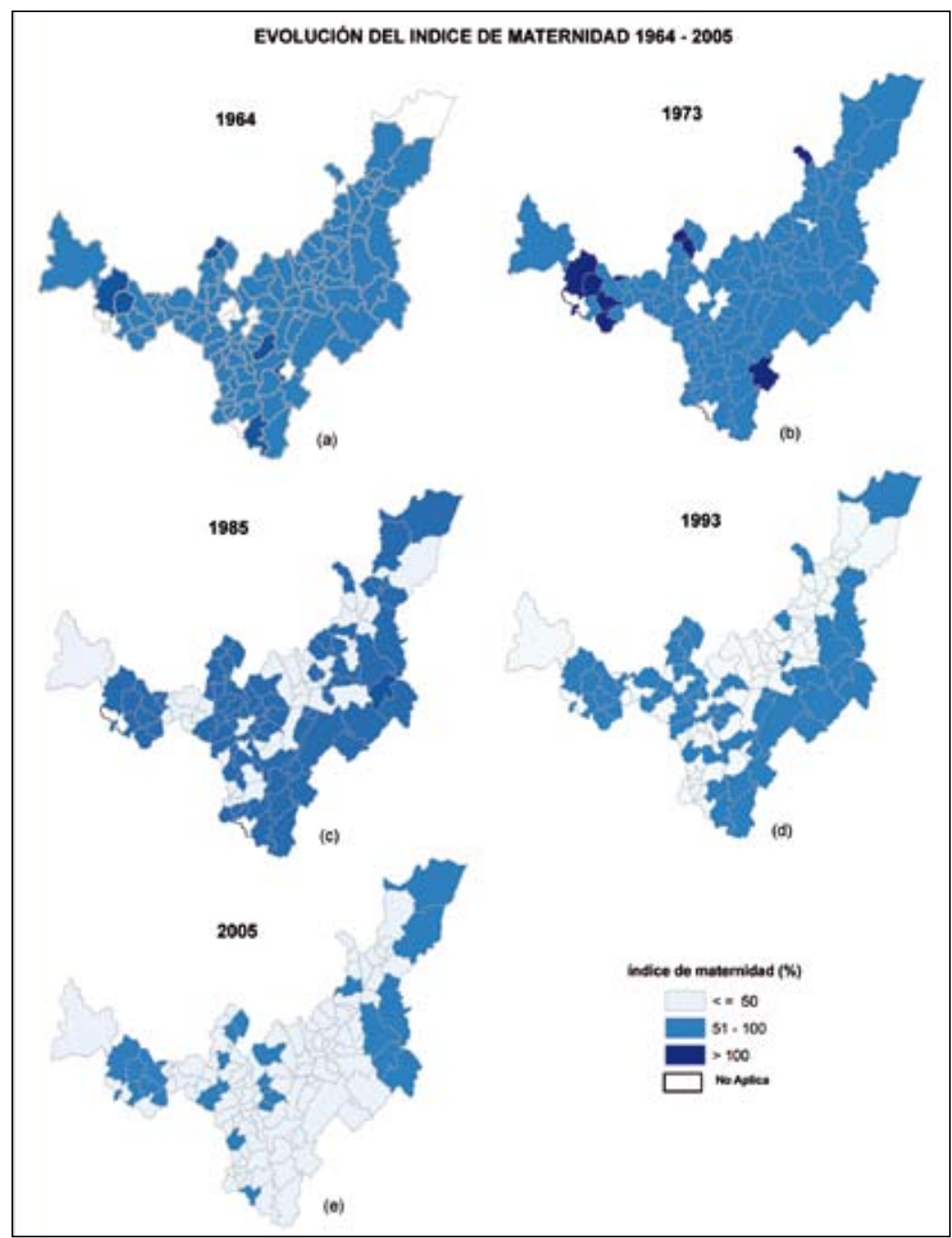

Fuente: elaboración propia. Cálculos basados en datos del DANE, censos 1964, 1973,1985, 1993, 2005. 
El índice de envejecimiento, definido como la relación entre la población vieja (mayor de 65 años) respecto a la población total, permite caracterizar el proceso de envejecimiento de una población. Aunque se considera que en los municipios boyacenses este índice aún no ha alcanzado límites que indiquen un envejecimiento inminente, sí ha tenido un ligero aumento, especialmente entre 1993 y 2005. En 1964 y 1973, la mayoría (91 \% y $84 \%$ ) de los municipios presentaban valores bajos (Tabla 6), aunque se observan algunos valores medios en enclaves territoriales aislados (Figuras 5a y 5b), pero a partir de 1985 estos enclaves comienzan a expandirse hasta el 2005 cuando se observa una distribución más homogénea de los valores medios (Figuras 5c, 5d, 5e), notándose que la mayoría de los enclaves de 1964 experimentan ahora valores altos. En ese sentido se hace necesario que los municipios de San Mateo, Guacamayas y Sativanorte, localizados en el norte del departamento; Guayatá, Somondoco, Sutatenza, Almeida, la Capilla, Pachavita y Chinavita ubicados al suroriente, y que hacen parte de dichos enclaves de valores altos, sean objeto de estudios que permitan conocer y prever las necesidades de salud, bienestar y vivienda, entre otras, de esa población vieja que está en aumento.
De igual manera, el índice de renovación de población activa IRPA, definido como la relación entre el tamaño de los grupos en edad en incorporarse al mercado laboral con aquellos en los que se produce la salida, pretende medir la capacidad de una población para sustituir a los individuos que se van jubilando, indicando la posible relación futura entre demanda y oferta de empleo. El IRPA ha sido fluctuante en los municipios del departamento, aunque en general presenta una tendencia hacia el aumento (Tabla 7), lo que significa que en la mayoría de los municipios hay población suficiente en edad de trabajar para remplazar a la población que está en etapa de jubilación. En 1964, la mayoría de los municipios experimentó un índice de IRPA bajo, aunque se observan enclaves territoriales con índices altos, especialmente en las zonas limítrofes del departamento (Figura 6a), los cuales se mantuvieron hasta 1993 en dicho rango (Figuras 6b, 6c, 6d). A partir de $1973 \mathrm{se}$ consolida la tendencia predominante de índices de IRPA medio, reafirmándose en el 2005 con el $93 \%$ de los municipios en dicho rango, mientras los municipios de los extremos oriental y occidental del departamento y los del corredor vial presentan valores altos (Figura 6e). De acuerdo con lo anterior, es imprescindi-

Tabla 6. Distribución porcentual de municipios según índice de envejecimiento 1964-2005

\begin{tabular}{|c|c|c|c|c|c|c|}
\hline \multirow{2}{*}{ Periodo } & \multicolumn{2}{|c|}{ Bajo } & \multicolumn{2}{c|}{ Medio } & \multicolumn{2}{c|}{ Alto } \\
\cline { 2 - 7 } & Num mpio & mpio (\%) & Num mpio & mpio (\%) & Num mpio & mpio(\%) \\
\hline 1964 & 100 & 91 & 10 & 9 & 0 & 0 \\
\hline 1973 & 96 & 84 & 18 & 16 & 0 & 0 \\
\hline 1985 & 55 & 45 & 66 & 55 & 0 & 0 \\
\hline 1993 & 32 & 26 & 91 & 74 & 0 & 0 \\
\hline 2005 & 6 & 5 & 107 & 87 & 10 & 8 \\
\hline
\end{tabular}

Fuente: elaboración propia. Cálculos basados en censos, DANE, 1964, 1973, 1985, 1993, 2005 
Figura 5. Evolución del índice de envejecimiento 1964-2005

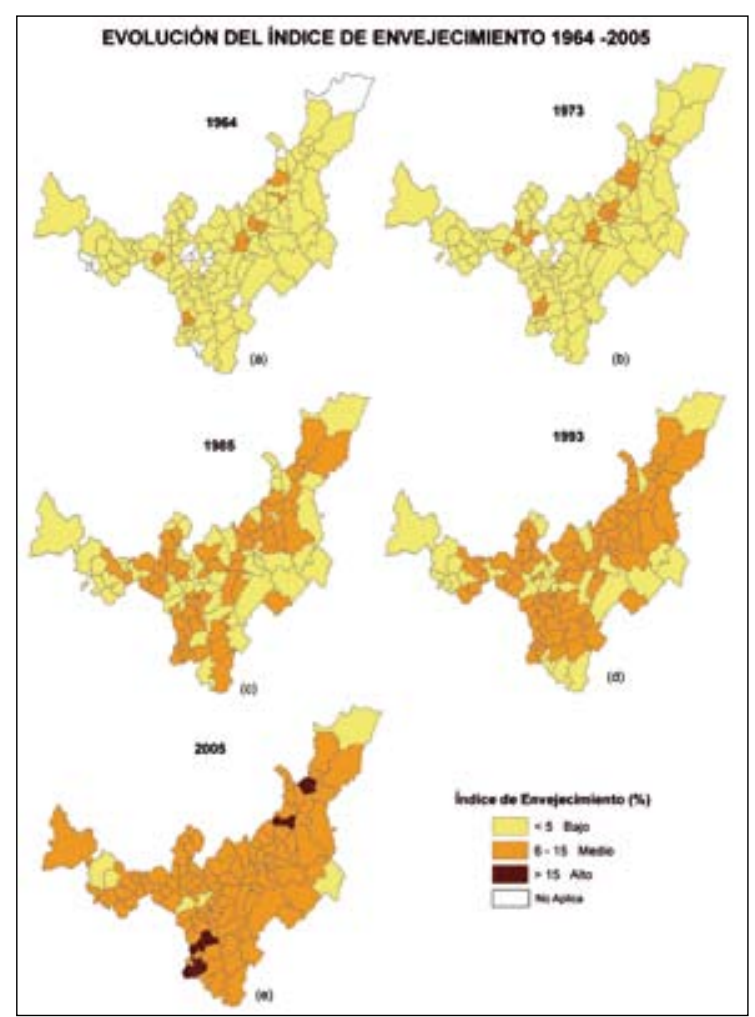

Fuente: elaboración propia. Cálculos basados en censos DANE, 1964, 1973, 1985, 1993, 2005.

ble que los municipios con valores altos, de investigaciones que permitan encontrar como Cubará, Puerto Boyacá, Tunja, el soluciones al inminente aumento en la tasa Espino, Cómbita y Samacá sean objeto de desempleo.

Tabla 7. Distribución porcentual de municipios según IRPA 1964 -2005

\begin{tabular}{|c|c|c|c|c|c|c|}
\hline \multirow{3}{*}{ Periodo } & \multicolumn{5}{|c|}{ Municipios según índice de Renovación de Población Activa } & \\
\hline & \multicolumn{2}{|c|}{ Bajo } & \multicolumn{2}{|c|}{ Medio } & \multicolumn{2}{|c|}{ Alto } \\
\hline & Num. Mpio & $\%$ Mpio & Num. Mpio & \% Mpio & $\begin{array}{l}\text { Num. } \\
\text { Mpio }\end{array}$ & \% Mpio \\
\hline 1964 & 84 & 76 & 0 & 0 & 26 & 24 \\
\hline 1973 & 1 & 1 & 94 & 82 & 19 & 17 \\
\hline 1985 & 2 & 2 & 91 & 75 & 28 & 23 \\
\hline 1993 & 1 & 1 & 97 & 79 & 25 & 20 \\
\hline 2005 & 3 & 2 & 114 & 93 & 6 & 5 \\
\hline
\end{tabular}

Fuente: elaboración propia 
Figura 6. Evolución del índice de renovación de población activa. Boyacá 1964-2005

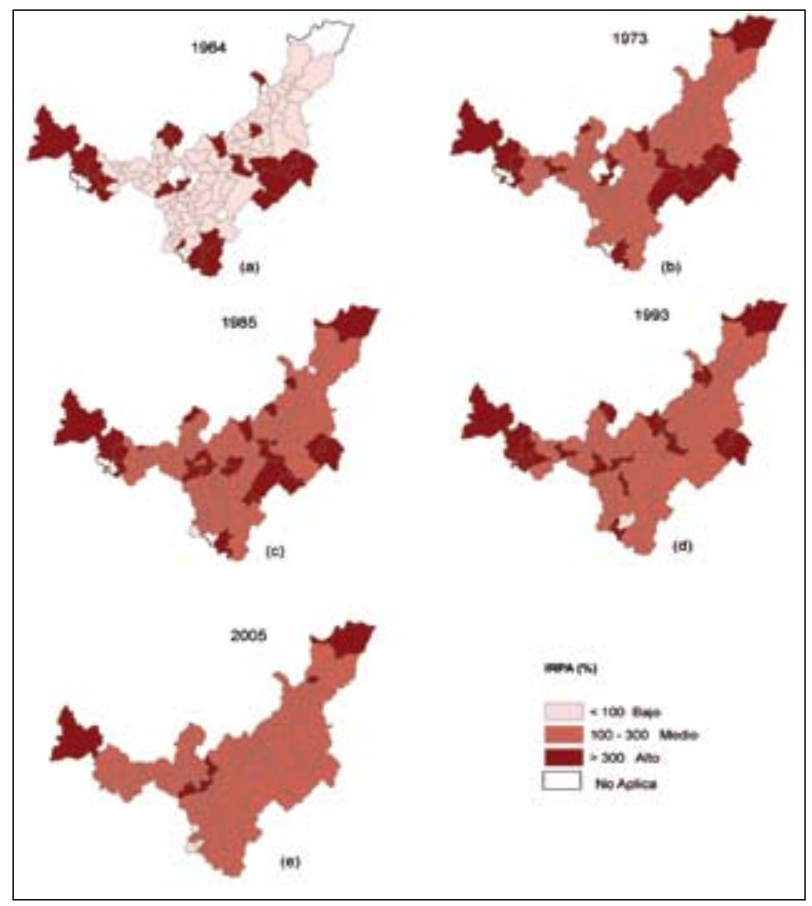

Fuente: elaboración propia. Cálculos basados en datos del DANE, Censos 1964, 1973,1985, 1993, 2005

Por otro lado, el índice de dependencia permite establecer la relación entre el grupo de población potencialmente activa y los grupos de individuos económicamente dependientes. Por tener un significado más económico, funciona como aproximación a la carga económica de las personas que están en edad de trabajar. En los municipios boyacenses, este índice no supera el $200 \%$, es decir que por cada persona en edad de trabajar, no alcanzan a existir dos personas en edad de dependencia económica. Éste índice presentaba en 1964 valores medios (mayores a 100 $\%$ ) en la mayoría de los municipios del departamento, notándose un patrón más o menos homogéneo (figura 7a). En 1973, los ámbitos de valores medios presentan una ligera contracción, en tanto que los valores bajos empiezan a expandirse por el territorio (Figura 7b), y en 1985 la expansión de los índices de valores medios es casi total, ya que solo el $8 \%$ de los municipios continúa manteniendo valores medios (Figura 7c). En 1993, el índice continúa con dicho comportamiento (Figura 7d) hasta el 2005 cuando el panorama es totalmente contrastante con el de 1964 (Figura 7e), ya que el 100 $\%$ de los municipios presenta índices de valores bajos (Tabla 8). Lo anterior señala de manera aproximada que la carga económica de la gente potencialmente productiva es baja. 
Figura 7. Evolución del índice de dependencia 1964-2005

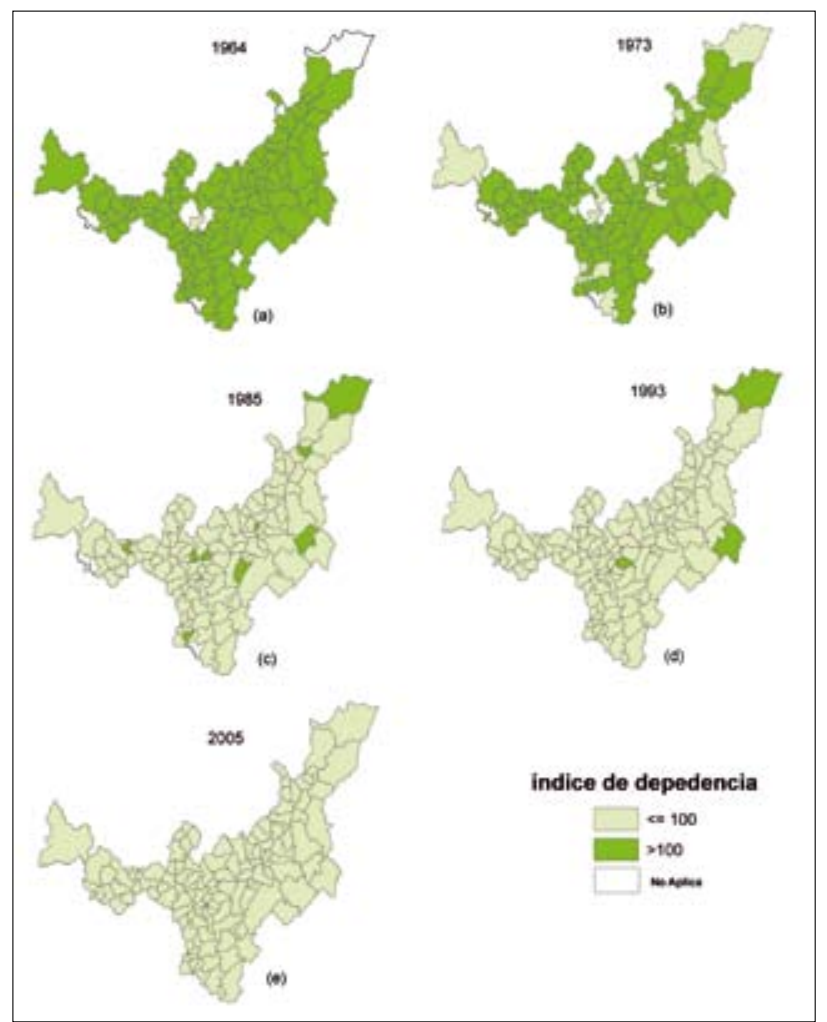

Fuente: elaboración propia. Cálculos basados en datos del DANE, censos 1964, 1973,1985, 1993, 2005

Tabla 8. Distribución porcentual de municipios según índice de dependencia 1964-2005

\begin{tabular}{|c|c|c|c|c|}
\hline \multirow{2}{*}{ Periodo } & \multicolumn{2}{|c|}{ Bajo } & \multicolumn{2}{c|}{ Medio } \\
\cline { 2 - 5 } & Num. Mpio & \% Mpio & Num. Mpio & $\%$ Mpio \\
\hline 1964 & 1 & 1 & 109 & 99 \\
\hline 1973 & 20 & 18 & 94 & 82 \\
\hline 1985 & 111 & 92 & 10 & 8 \\
\hline 1993 & 120 & 98 & 3 & 2 \\
\hline 2005 & 123 & 100 & 0 & 0 \\
\hline
\end{tabular}

Fuente: elaboración propia. 


\section{Conclusiones}

La dinámica demográfica municipal de Boyacá pasó de un perfil demográfico expansivo en 1964 a un perfil regresivo en el 2005. En forma general, todos los índices reflejan que hay una tendencia al decrecimiento poblacional, con un perfil regresivo debido a la disminución constante de población infantil, a la leve constricción de población de adultos jóvenes y a un ligero aumento de población vieja. Aunque en unos pocos municipios se evidencia un mayor envejecimiento, la dependencia económica en el departamento es baja y la capacidad de remplazar la población que está en edad de jubilarse es media.

De acuerdo con la distribución espacial del comportamiento de estos indicadores, se puede concluir que los municipios de Boyacá experimentan diferentes dinámicas, pero especialmente los municipios limítrofes de la zona oriental y el municipio de Puerto Boyacá, que presentan diferencias en todos los índices, con excepción del índice de envejecimiento.

Con base en el comportamiento de estos índices demográficos municipales es necesario plantear políticas regionales enfocadas al nivel municipal en temas de desempleo, en los municipios de Cubará, Puerto Boyacá, Tunja, el Espino, Cómbita y Samacá. Así mismo, se requiere investigar las necesidades de vivienda y salud de la población vieja en los municipios de San Mateo, Guacamayas y Sativanorte, localizados en el norte del departamento; Guayatá, Somondoco, Sutatenza, Almeida, la Capilla, Pachavita y Chinavita ubicados al suroriente, los cuales hacen parte de enclaves territoriales con valores altos del índice de envejecimiento. Por otro lado están el índice de tendencia y de maternidad que en la mayoría de los municipios señalan un posible decrecimiento demográfico, por lo que es importante estudiar sus causas y emprender acciones pertinentes a fin de evitar un futuro despoblamiento. 


\section{Literatura citada}

Flórez, C. E. (2000). Las transformaciones sociodemográficas en Colombia durante el Siglo XX. Bogotá: Banco de la Republica, Tercer Mundo.

Martínez, C. (2006). Las migraciones internas en Colombia. Bogotá: Universidad Externado de Colombia.

Departamento Administrativo Nacional de Estadística DANE. (1964). XIII Censo Nacional de población. Bogotá: Imprenta Nacional.

Departamento Administrativo Nacional de Estadística DANE. (1973). XIV Censo Nacional de Población y III de Vivienda. Bogotá: Imprenta Nacional

Departamento Administrativo Nacional de Estadística DANE. (1985). XV Censo Nacional de Población y IV de Vivienda. Bogotá: Printer Colombiana.

Departamento Administrativo Nacional de Estadística DANE. (1993). XVI Censo Nacional de Población y V de Vivienda. Bogotá. DANE

Departamento Administrativo Nacional de Estadística DANE. (2005). XVII Censo Nacional de Población y VI de Vivienda. Recuperado en abril, 2009, de http://190.25.231.242/cgibin/RpWebEngine.exe/PortalAction?\&MODE =M AIN\&BASE $=$ CG2005BASICO\&MAIN $=$ WebServerMain.$i n l$

Martonne, E. (1934). Traité de geographie physique. Paris: s.n.

Smith, D. (1980). Geografía humana. Barcelona: Oikos.

Vinuesa, J. \& Francisco, Z. (1997). Demografía, análisis y proyecciones. Madrid: Síntesis. 